The Rate of Convergence of the Modified Method of Characteristics for Linear Advection Equations in One Dimension

by

\author{
C.N. Dawson \\ T.F. Dupont \\ M.F. Wheeler
}

Technical Report 88-3, March 1988 



\title{
The Rate of Convergence of the Modified Method of Characteristics for Linear Advection Equations in One Dimension
}

\author{
Clint N. Dawson ${ }^{1}$, Todd F. Dupont ${ }^{2}$, and Mary F. Wheeler ${ }^{1}$
}

\section{Introduction}

In a recent paper by Russell and two of the authors [2], it was demonstrated that the error for the modified method of characteristics (MMOC) applied to periodic linear advection problems in $n$ dimensions is at worst $O\left(h^{r}+\Delta t\right)$ in $L^{2}$ at each time level. Here $r=\min (s, k)$, where $k$ is the degree of the piecewise polynomial approximating space and $s$ is related to the smoothness of the solution. Thus, a suboptimal rate of convergence in $L^{2}$ was derived; the rate is suboptimal in the sense that the power of $h$ is one lower than is possible for a best approximation.

In this paper, we show that in general the rate of convergence is suboptimal, but that it is optimal in at least one case. We consider the simple one-dimensional linear advection equation

$$
\left\{\begin{aligned}
u_{t}+u_{x}=f, & & x \in(0,1) \equiv I, \\
u(x, 0)=u^{0}(x), & & x \in I
\end{aligned}\right.
$$

with periodic initial and boundary conditions, i.e., $u^{0}(0)=u^{0}(1)$ and $u(0, t)=u(1, t)$. In particular, we consider a finite element MMOC approximation to (1.1) with continuous piecewise linear and continuous piecewise quadratic approximating spaces. In the first case (piecewise linears), we show that under certain assumptions on the smoothness of $u$ and assuming $\Delta t=\mathcal{O}\left(h^{2}\right)$, one actually has optimality in $L^{2}$, thus, the $L^{2}$ error is $\mathcal{O}\left(h^{2}\right)$. For the second case (piecewise quadratics), assuming $\Delta t=o\left(h^{2}\right)$, we construct an example where the $L^{2}$ error is at best $\mathcal{O}\left(h^{2}\right)$, thus, a suboptimal order of convergence holds here.

The nature of these results and the tools used are similar to the results and tools in [3] where the rate of convergence of the continuous-time Galerkin method was studied.

\footnotetext{
${ }^{1}$ Dept. of Mathematical Sciences, Rice University

${ }^{2}$ Dept. of Computer Science, University of Chicago
} 
Our result for piecewise quadratics can be used to see that the rate of convergence of the continuous-time Galerkin method is suboptimal in this case; just take $\Delta t$ to zero.

The remainder of this paper is divided into five sections. In the next section, we establish notation, discuss the MMOC and its application to (1.1), and state the two major results of this paper. The first major result, an optimal order $L^{2}$ error estimate for the case of a continuous piecewise linear approximating space, is derived in Section 3. The second major result is derived in Section 4; we show by construction that for the case of continuous piecewise quadratic approximation the rate in $L^{2}$ is suboptimal in space. Technical lemmas used to derive the results of Sections 3 and 4 are proved in Section 5. Finally, in Section 6, we verify these theoretical results numerically by calculating experimental $L^{2}$ convergence rates for a smooth test problem.

\section{Notation and Preliminaries}

Let $L^{2}(I), L^{\infty}(I)$, and $H^{k}(I)$ denote the standard Sobolev spaces defined on the unit interval. Let $\tilde{L}^{2}$ denote the set of functions defined on $\mathbf{R}$ which are one-periodic and whose restrictions to $I$ are on $L^{2}(I)$. Adopt a similar definition for $\tilde{H}^{k}$. For $u$ an element of $\tilde{L}^{2}$ let $\|u\|=\|u\|_{L^{2}(I)}$, and for $u \in \tilde{H}^{k},\|u\|_{k}=\|u\|_{H^{k}(I)}$.

If $\mathcal{X}$ is a normed space with norm $\|\cdot\|_{\mathcal{X}}$ and $\phi:[0, T] \rightarrow \mathcal{X}$, let

$$
\|\phi\|_{L^{\infty}(\mathcal{X})}=\sup _{0 \leq t \leq T}\|\phi(t)\|_{\mathcal{X}}
$$

For $M_{h}$ a finite-dimensional subspace of $\tilde{H}^{1}$, the finite element MMOC is a map $U:\left\{0=t^{0}, t^{1}, \ldots, t^{N}=T\right\} \rightarrow M_{h}$ defined by

$$
\left\{\begin{array}{l}
\left(\frac{U^{n+1}-\hat{U}^{n}}{\Delta t}, v\right)=\left(f^{n+1}, v\right), \quad v \in M_{h} \\
U^{0}=\tilde{U}^{0}
\end{array}\right.
$$

Here $(\cdot, \cdot)$ denotes the $L^{2}$ inner product on $I, f^{n}(x)=f\left(x, t^{n}\right), U^{n}(x)=U\left(x, t^{n}\right)$, where $t^{n}=n \Delta t, n=0,1, \ldots, N$,

$$
\hat{U}^{n}(x)=U^{n}(x-\Delta t)
$$

and $\tilde{U}^{0}$ is the $L^{2}$ projection of $u^{0}$ into $M_{h}$. (For application and analysis of the MMOC to more complicated problems, see [4], [5], [6], [7].)

We note that since the true solution satisfies

$$
u\left(x, t^{n+1}\right)=u\left(x-\Delta t, t^{n}\right)+\int_{0}^{\Delta t} f\left(x-\Delta t+s, t^{n}+s\right) d s
$$

we have

$$
\left(\frac{u^{n+1}-\hat{u}^{n}}{\Delta t}, v\right)=\left(f^{n+1}+\rho^{n}, v\right), \quad v \in M_{h}
$$


where $\rho_{n}=\mathcal{O}(\Delta t)$ provided $f_{\tau} \in L^{\infty}\left(L^{2}\right)$, where $\tau$ is the characteristic direction.

Let $J$ be a positive integer, $h=1 / J$, and for $j=1, \ldots, J$,

$$
\begin{aligned}
x_{j} & =j h, \\
X_{j} & =\left[x_{j-1}, x_{j}\right], \\
x_{j+1 / 2} & =(j+1 / 2) h .
\end{aligned}
$$

Denote by $\tilde{M}_{0}(1, h)$ the set of functions $v \in \mathcal{C}^{0}(I)$ with $v$ linear on each $x_{j}$, and $v(0)=$ $v(1)$. Moreover, denote by $\tilde{M}_{0}(2, h)$ the set of functions $v \in \mathcal{C}^{0}(I), v$ quadratic on $X_{j}$, and $v(0)=v(1)$. We now study convergence of the MMOC with $M_{h}=\tilde{M}_{0}(1, h)$ and $M_{h}=\tilde{M}_{0}(2, h)$.

The main results of this paper can be stated as follows.

Theorem 1 Let $\Delta t=\mathcal{O}\left(h^{2}\right)$, and assume $u$ is three times continuously differentiable, then

$$
\max _{0 \leq n \leq N}\left\|u^{n}-U^{n}\right\|_{L^{2}}=\mathcal{O}\left(h^{2}\right)
$$

Theorem 2 Let $\Delta t=o\left(h^{2}\right)$ and assume $u$ is four times continuously differentiable, then

$$
\max _{0 \leq n \leq N}\left\|u^{n}-U^{n}\right\|_{L^{2}}=\mathcal{O}\left(h^{2}\right) \text {. }
$$

In addition, if $u_{x x x}$ is not identically zero, there exists $C_{0}>0$ independent of $h$ and $\mu^{n} \in \tilde{M}_{0}(2, h)$ with $\max _{n}\left\|\mu^{n}\right\| \geq C_{0}$, and

$$
\left\|u^{n}-U^{n}+h^{2} \mu^{n}\right\|_{L^{2}}=\mathcal{O}\left(h^{3}\right) .
$$

Consequently, the estimate (2.4) is sharp.

In the following analysis, $C$ represents a generic constant independent of $h$ and $\Delta t$.

\section{Proof of Theorem 1.}

Let $M_{h}=\tilde{M}_{0}(1, h)$, and for each $t \in(0, T]$, let $\tilde{U}(t)$ be the $\tilde{L}^{2}$ projection of $u(t)$ into $M_{h}$, i.e.

$$
(\tilde{U}(t)-u(t), v)=0, \quad v \in M_{h} .
$$

Then, setting $\zeta=U-\tilde{U}, \xi=u-\tilde{U}$, we have by (2.1), (2.3), and (3.1)

$$
\left(\frac{\zeta^{n+1}-\hat{\zeta}^{n}}{\Delta t}, v\right)=\left(\frac{\xi^{n}-\hat{\xi}^{n}}{\Delta t}+\rho^{n}, v\right), \quad v \in M_{h} .
$$


Noting by periodicity that $\left\|\hat{\zeta}^{n}\right\|=\left\|\zeta^{n}\right\|$, and setting $v=\zeta^{n+1}$ in (3.2) we obtain

$$
\left\|\zeta^{n+1}\right\|^{2}-\left\|\zeta^{n}\right\|^{2}+\left\|\zeta^{n+1}-\hat{\zeta}^{n}\right\|^{2}=2\left(\xi^{n}-\hat{\xi}^{n}+\Delta t \rho^{n}, \zeta^{n+1}\right) .
$$

A suboptimal $L^{2}$ estimate for $\zeta$ can be obtained as in [2] by noting that

$$
\left(\xi^{n}-\hat{\xi}^{n}+\Delta t \rho^{n}, \zeta^{n+1}\right) \leq C \Delta t\left(\left\|\xi_{x}^{n}\right\|^{2}+\left\|\rho^{n}\right\|^{2}+\left\|\zeta^{n+1}\right\|^{2}\right) .
$$

Moreover, by approximation theory

$$
\left\|\xi_{x}^{n}\right\| \leq C h\left\|u^{n}\right\|_{2} \text {. }
$$

Thus, substituting (3.4) and (3.5) into (3.3) and summing on $n, n=0, \ldots, N-1$ we find that

$$
\left\|\zeta^{N}\right\|^{2} \leq C_{u}\left(h^{2}+(\Delta t)^{2}\right)+C \sum_{n=0}^{N-1}\left\|\zeta^{n+1}\right\|^{2} \Delta t
$$

where $C_{u}$ depends on derivatives of $u$ through second order. By the discrete Gronwall Lemma,

$$
\max _{0 \leq n \leq N}\left\|\zeta^{n}\right\| \leq C(h+\Delta t) \text {. }
$$

Combining (3.6) with the well-known estimate

$$
\|\xi\|_{L^{\infty}\left(L^{2}\right)} \leq C h^{2}\|u\|_{L^{\infty}\left(H^{2}\right)},
$$

we have by the triangle inequality

$$
\max _{0 \leq n \leq N}\left\|u^{n}-U^{n}\right\| \leq C(h+\Delta t) .
$$

We can see by the above argument that obtaining a "better" $L^{2}$ estimate requires more careful treatment of the term

$$
\left(\xi^{n}-\hat{\xi}^{n}, \zeta^{n+1}\right)
$$

Thus, we examine this term more closely.

We now state a lemma concerning the term (3.9), whose proof we defer to Section 5 .

Lemma 1 Assume the hypothesis of Theorem 1 holds, then

$$
\left|\left(\frac{\xi^{n}-\hat{\xi}^{n}}{\Delta t}, \zeta^{n+1}\right)\right| \leq C\left(h^{4}+\left\|\zeta^{n+1}\right\|^{2}\right) .
$$

Substituting (3.10) into (3.3), assuming $\Delta t=\mathcal{O}\left(h^{2}\right)$, we find

$$
\frac{1}{\Delta t}\left\|\zeta^{n+1}\right\|^{2} \leq \frac{1}{\Delta t}\left\|\zeta^{n}\right\|^{2}+C h^{4}+\left\|\zeta^{n+1}\right\|^{2} .
$$

Summing above on $n$ and applying the discrete Gronwall Lemma proves Theorem 1. 


\section{Proof of Theorem 2.}

As in the proof of Theorem 1 with $\xi=u-\tilde{U}$ and $\zeta=U-\tilde{U}$, we obtain

$$
\left(\frac{\zeta^{n+1}-\hat{\zeta}^{n}}{\Delta t}, \chi\right)=\left(\frac{\xi^{n}-\hat{\xi}^{n}}{\Delta t}+\rho^{n}, \chi\right), \quad \chi \in \tilde{M}_{0}(2, h) .
$$

Setting $\chi=\zeta^{n+1}$, we have

$$
\frac{1}{\Delta t}\left\|\zeta^{n+1}\right\|^{2} \leq \frac{1}{\Delta t}\left\|\zeta^{n}\right\|^{2}+\left(\frac{\xi^{n}-\hat{\xi}^{n}}{\Delta t}+\rho^{n}, \zeta^{n+1}\right)
$$

Just as in the derivation of the optimal rate in the proof of Theorem 1 we get that

$$
\frac{1}{\Delta t}\left\|\zeta^{n+1}\right\|^{2} \leq \frac{1}{\Delta t}\left\|\zeta^{n}\right\|^{2}+C\left(h^{4}+\left\|\zeta^{n+1}\right\|^{2}\right)
$$

Here we have also used the fact that $\Delta t=o\left(h^{2}\right)$. Summing (4.2) on $n, n=0,1, \ldots, N$ and applying Gronwall's Lemma we obtain (2.5).

In Lemma 4 below we construct $\mu^{n} \in \tilde{M}_{0}(2, h)$ satisfying $\max _{n}\left\|\mu^{n}\right\| \geq C_{0}>0$ and

$$
\left\|\zeta^{n}+h^{2} \mu^{n}\right\|=\mathcal{O}\left(h^{3}\right)
$$

Inequality (2.4) follows immediately using approximation theory.

\section{Lemmas.}

\section{V.1 Proof of Lemma 1.}

Let $\mathcal{V}=\left\{v_{0}(x), v_{1}(x), \ldots, v_{J-1}(x)\right\}$ be the set of 1-periodic "hat" functions defined over the mesh $\left\{x_{j}\right\}$. That is, $v_{j} \in \tilde{M}_{0}(1, h)$ and

$$
v_{j}\left(x_{k}\right)= \begin{cases}1, & j=k \\ 0, & 0 \leq k<J, k \neq j\end{cases}
$$

Then $\mathcal{V}$ is a basis for $\tilde{M}_{0}(1, h)$ and

$$
\zeta^{n}(x)=\sum_{j=0}^{J-1} \zeta_{j}^{n} v_{j}(x)
$$

where

$$
\zeta_{j}^{n}=\zeta^{n}\left(x_{j}\right)
$$

It is easily seen that if

$$
\chi(x)=\sum_{j=0}^{J-1} \chi_{j} v_{j}(x)
$$


and

$$
\|\chi\|_{h}^{2}=\sum_{j=0}^{J-1} \chi_{j}^{2} h
$$

then

$$
\frac{1}{3}\|\chi\|_{h}^{2} \leq\|\chi\|^{2} \leq\|\chi\|_{h}^{2}
$$

Thus $\|\cdot\|_{h}$ and $\|\cdot\|$ define equivalent norms on $\tilde{M}_{0}(1, h)$.

Suppressing time-dependence momentarily, we express $\xi$ as

$$
\xi(x)=\psi(x)+g(x)
$$

where on each $X_{j+1}$

$$
\psi(x)=\frac{1}{2} u_{x x}\left(x_{j+\frac{1}{2}}\right)\left(x-y_{j 1}\right)\left(x-y_{j 2}\right)
$$

with $y_{j 1}$ and $y_{j 2}$ being the two Gauss points on $X_{j+1}$. It then follows [3] that on each $X_{j+1}$

$$
|g(x)|+h\left|g^{\prime}(x)\right|=\mathcal{O}\left(h^{3}\right) ;
$$

i.e., $\psi$ is the leading term in the error in the $L^{2}$ projection.

This expression is used to show that $\left(\xi_{x}, v_{j}\right)$ is smaller than is apparent at first glance. Specifically, integration by parts shows that

$$
\begin{gathered}
\left(\psi^{\prime}, v_{j}\right)=\frac{1}{2}\left(x_{j}-y_{j 1}\right)\left(x_{j}-y_{j 2}\right)\left(u_{x x}\left(x_{j-\frac{1}{2}}\right)-u_{x x}\left(x_{j+\frac{1}{2}}\right)\right) \\
+\left(\dot{\psi}, v_{j}^{\prime}\right) .
\end{gathered}
$$

The last integral is zero since $\psi$ vanishes at the Gauss points. Combining this with (5.7) we see that

$$
\left(\xi_{x}, v_{j}\right)=\mathcal{O}\left(h^{3}\right)
$$

Next, let

$$
\xi(x)-\xi(x-\Delta t)=\Delta t \xi_{x}(x)+\eta(x)
$$

Then for each $j$

$$
\eta(x)= \begin{cases}\mathcal{O}\left(\Delta t^{2}\right), & \text { on }\left(x_{j}+\Delta t, x_{j+1}\right) \\ \mathcal{O}(h \Delta t), & \text { on }\left(x_{j}, x_{j}+\Delta t\right)\end{cases}
$$

The first bound holds because $\xi_{x x}=u_{x x}$ on $\left(x_{j}, x_{j+1}\right)$; the second holds since $\xi_{x}=\mathcal{O}(h)$ and

$$
\xi(x)-\xi(x-\Delta t)=\int_{x-\Delta t}^{x} \xi_{x}(s) d s
$$

From (5.11) we see that

$$
\left(\eta, v_{j}\right)=\mathcal{O}\left(h \Delta t^{2}\right)
$$


Thus from (5.9), (5.10) and (5.12) we see that

$$
\begin{aligned}
\left(\xi-\hat{\xi}, v_{j}\right) & =\mathcal{O}\left(h^{3} \Delta t+h \Delta t^{2}\right) \\
& =\mathcal{O}\left(h^{3} \Delta t\right) .
\end{aligned}
$$

Hence,

$$
\begin{aligned}
\left(\xi^{n}-\hat{\xi}^{n}, \zeta^{n+1}\right) & =\sum_{j}\left(\xi^{n}-\hat{\xi}^{n}, v_{j}\right) \zeta_{j}^{n+1} \\
& \leq C \Delta t \sum_{j} h^{2}\left|\zeta_{j}^{n+1}\right| h \\
& \leq C \Delta t\left(h^{4}+\left\|\zeta^{n+1}\right\|^{2}\right) .
\end{aligned}
$$

Here we used (5.4). This completes the proof of Lemma 1.

\section{V.2 Quadratic Case Truncation Error}

Let $v_{j}, j=0, \ldots, J-1$ be the "hat" functions defined by (5.1) and let $b_{j}, j=$ $0, \ldots, J-1$ be the "bump" functions given by

$$
b_{j}(x)=\left\{\begin{array}{cl}
\frac{\left(x-x_{j}\right)\left(x_{j+1}-x\right)}{h^{2}}, & x_{j} \leq x \leq x_{j+1}, \\
0, & \text { otherwise }
\end{array}\right.
$$

Then, $\left\{v_{j}\right\} \cup\left\{b_{j}\right\}$ is a basis for $\tilde{M}_{0}(2, h)$. We now show that

$$
\left(\frac{\xi^{n}-\hat{\xi}^{n}}{\Delta t}, v_{j}\right)=\mathcal{O}\left(h^{4}\right)
$$

and

$$
\left(\frac{\xi^{n}-\hat{\xi}^{n}}{\Delta t}, b_{j}\right)=-\frac{1}{360} u_{x x x}\left(x_{j}, t^{n}\right) h^{3}+\text { higher order terms. }
$$

Again suppressing time-dependence momentarily, we have

$$
\frac{\xi(x)-\hat{\xi}(x)}{\Delta t}=\xi_{x}(x)+e(x),
$$

where $e(x)=\bar{\xi}_{x}(x)-\xi_{x}(x)$ and $\bar{\xi}_{x}$ is the average value of $\xi_{x}$ over the interval $[x-\Delta t, x]$. Using Lemmas 2 and 3 , the results (5.15) and (5.16) follow.

Lemma 2 Let $u$ be $\mathcal{C}^{4}$ and 1-periodic. Then

$$
\left(\xi_{x}, v_{j}\right)=\mathcal{O}\left(h^{4}\right)
$$

and

$$
\left(\xi_{x}, b_{j}\right)=-\frac{1}{360} u_{x x x}\left(x_{j}\right) h^{3}+\mathcal{O}\left(h^{4}\right),
$$

where $v_{j}$ and $b_{j}$ are defined by (5.1) and (5.14). 
Proof: Let $I u$ denote the $\mathcal{C}^{0}$ piecewise quadratic interpolant of $u$ on $X_{j+1}$ defined by interpolation of $u$ at $x_{j}, x_{j+\frac{1}{2}}$ and $x_{j+1}$. Using a Hermite-Birkhoff cubic interpolant which in addition to the conditions for $I u$ matches $u_{x x x}$ at $x_{j+\frac{1}{2}}$, and applying the Bramble-Hilbert Lemma [1], we have on $X_{j+1}$

$$
\begin{aligned}
(u-I u)(x) & =\frac{1}{6} u_{x x x}\left(x_{j+\frac{1}{2}}\right)\left(x-x_{j}\right)\left(x-x_{j+\frac{1}{2}}\right)\left(x-x_{j+1}\right)+g(x) \\
& \equiv \psi(x)+g(x),
\end{aligned}
$$

where $|g(x)|+h\left|g^{\prime}(x)\right|=\mathcal{O}\left(h^{4}\right)$. Note that

$$
\left(\psi, v_{j}\right)=\mathcal{O}\left(h^{5}\right)
$$

and

$$
\left(\psi, b_{j}\right)=0 .
$$

Now $\xi(x)=(u-I u)+(I u-\tilde{U})$. Thus, if follows easily that

$$
\xi=\psi+\mathcal{O}\left(h^{4}\right)
$$

and

$$
\xi_{x}=\psi^{\prime}+\mathcal{O}\left(h^{3}\right) .
$$

Thus, the leading order term in the expression for $\xi_{x}(x)$ is $\psi^{\prime}(x)$. The lemma now follows since

$$
\left(\psi^{\prime}, b_{j}\right)=-\frac{1}{360} u_{x x x}\left(x_{j+\frac{1}{2}}\right) h^{3} .
$$

Lemma 3 Let $\bar{\xi}_{x}(x)$ denote the average value of $\xi_{x}$ over the interval $[x-\Delta t, x]$. Assume $\Delta t=o\left(h^{2}\right)$. Then $e=\bar{\xi}_{x}-\xi_{x}$ is $\mathcal{O}\left(h^{3}\right)$.

Proof. In the proof of Lemma 2, Equation (5.18), we showed that

$$
\xi(x)=\psi(x)+\text { higher order terms }
$$

on $X_{j}$, where $\psi$ is given by $(5.17)$. Now

$$
\left|\psi^{\prime}\left(x_{j}+0\right)-\psi^{\prime}\left(x_{j}-0\right)\right| \leq C h^{3},
$$

which implies that

$$
\left|\xi_{x}\left(x_{j}+0\right)-\xi_{x}\left(x_{j}-0\right)\right| \leq C h^{3}
$$

Moreover

$$
\bar{\xi}_{x}(x)=\frac{1}{\Delta t} \int_{x-\Delta t}^{x} \xi_{x}(s) d s=\xi_{x}(x)+\frac{1}{\Delta t} \int_{x-\Delta t}^{x}\left(\xi_{x}(s)-\xi_{x}(x)\right) d s .
$$

By Taylor expansion and (5.19)

$$
\begin{aligned}
\left|\xi_{x}(s)-\xi_{x}(x)\right| & \leq C h|x-s|+C h^{3} \\
& \leq C h \Delta t+C h^{3}
\end{aligned}
$$


Hence, by (5.20), (5.21), and the assumption on $\Delta t$

$$
\begin{aligned}
|e(x)| & =\left|\vec{\xi}_{x}(x)-\xi_{x}(x)\right| \\
& \leq C h \Delta t \\
& \leq C h^{3} .
\end{aligned}
$$

\section{V.3 Lemma 4}

Lemma 4 Assume that $u_{x x x}$ is not identically zero. Let

$$
\mu^{n}(x)=\sum_{j} r\left(x_{j}, t^{n}\right) w_{j}(x)
$$

where $w_{j}=-\frac{1}{3} v_{j}+b_{j}+b_{j-1}$. Assume $\Delta t=o\left(h^{2}\right)$ and that the coefficients $r(x, t)$ satisfy the first order equation

$$
r_{t}-10 r_{x}=-\frac{1}{4} u_{x x x},
$$

with $r(x, 0) \equiv 0$. Then, there exists $C_{0}>0$, independent of $h$, such that

$$
\max _{0 \leq n \leq N}\left\|\mu^{n}\right\| \geq C_{0}
$$

and

$$
\left\|\zeta^{n}+h^{2} \mu^{n}\right\|=\mathcal{O}\left(h^{3}\right)
$$

Proof: It is clear that $r$ is a function that is not identically zero and does not depend on $h$. The size of $\mu$ is determined by the size of $r$. In fact,

$$
\left\|\mu^{n}\right\|^{2} \geq \frac{1}{45} \sum_{j}\left|r\left(x_{j}, t^{n}\right)\right|^{2} h .
$$

Thus, the existence of $C_{0}>0$ independent of $h$ is established.

Let $\lambda=\frac{\Delta t}{h}$. A calculation yields

$$
\begin{aligned}
\frac{6}{h}\left(\mu^{n+1}-\hat{\mu}^{n}, v_{j}\right) \\
=-\frac{1}{3}\left[r_{j-1}^{n+1}+4 r_{j}^{n+1}+r_{j+1}^{n+1}-\left((1+3 \lambda) r_{j-1}^{n}+4 r_{j}^{n}+(1-3 \lambda) r_{j+1}^{n}\right)\right] \\
\quad+\frac{1}{2}\left[r_{j-1}^{n+1}+2 r_{j}^{n+1}+r_{j+1}^{n+1}-\left((1+2 \lambda)\left(r_{j-1}^{n}+r_{j}^{n}\right)+(1-2 \lambda)\left(r_{j}^{n}+r_{j+1}^{n}\right)\right)\right] \\
\quad+o(\Delta t) \\
=o\left(h^{2}\right) .
\end{aligned}
$$


and

$$
\begin{aligned}
\frac{60}{h}( & \left.\mu^{n+1}-\hat{\mu}^{n}, b_{j}\right) \\
= & -\frac{5}{3}\left[r_{j}^{n+1}+r_{j+1}^{n+1}-\left(r_{j}^{n}+r_{j+1}^{n}\right)+2 \lambda\left(r_{j+1}^{n}-r_{j}^{n}\right)\right] \\
& +2\left[r_{j}^{n+1}+r_{j+1}^{n+1}-\left(r_{j}^{n}+r_{j+1}^{n}\right)\right]+o(\Delta t) \\
= & \Delta t\left[\frac{1}{3} r_{t}\left(x_{j}, t^{n}\right)-\frac{10}{3} r_{x}\left(x_{j}, t^{n}\right)\right]+\mathcal{O}(h \Delta t)+o(\Delta t) .
\end{aligned}
$$

Thus,

$$
\left(\frac{\mu^{n+1}-\hat{\mu}^{n}}{\Delta t}, b_{j}\right)=-\frac{h}{360} u_{x x x}\left(x_{j}, t^{n}\right)+o(h) .
$$

Letting $\phi^{n}=\zeta^{n}+h^{2} \mu^{n}$ and using Lemmas 2 and 3, (5.22) and (5.23), we note that

$$
\left(\frac{\phi^{n+1}-\hat{\phi}^{n}}{\Delta t}, \phi^{n+1}\right)=\mathcal{O}\left(h^{3}\left\|\phi^{n+1}\right\|\right) .
$$

Thus, by Gronwall's Lemma

$$
\left\|\phi^{n}\right\|=\mathcal{O}\left(h^{3}\right)
$$

and the proof is complete.

\section{Experimental results}

In this section, we verify experimentally the theoretical results of the previous sections.

Experimental rates of convergence for the MMOC with piecewise linear and piecewise quadratic approximating spaces were computed for the test problem

$$
\left\{\begin{array}{rlrl}
u_{t}+u_{x} & =0, & & x \in(0,1) \equiv I, \\
u^{0}(x) & =\sin (2 \pi x), & x \in I,
\end{array}\right.
$$

which has solution $u(x, t)=\sin (2 \pi(x-t))$. Discrete $L^{2}$ errors were calculated at $t=$ $1 / 16$ for $h^{-1}=2^{k}, k=3, \ldots, 6$. For the case $M_{h}=\tilde{M}_{0}(1, h), \Delta t=h^{2}$ and the error was calculated by the trapezoidal rule of integration. For the case $M_{h}=\tilde{M}_{0}(2, h)$, $\Delta t=h^{3}$ and Simpson's rule was used. A least squares fit was used to calculated the experimental rate of convergence. Table 1 below verifies that in both the linear and quadratic cases, $h^{2}$ convergence was observed.

We would also like to report that, in the linear case with $f \equiv 0$, one obtains superconvergence of $h^{4}$ at the nodes, provided that the interpolant is used as the initial condition. This result can be verified theoretically, however, since it is very specialized, the details are not included here.

Moreover, it is clear from the proof of Theorem 2 that in the $\tilde{M}_{0}(2, h)$ case the convergence at the Gauss points $y_{j 1}$ and $y_{j 2}$ is $\mathcal{O}\left(h^{3}\right)$ since $\mu$ vanishes to the next higher order at these points. This was observed in our experiments. 


\begin{tabular}{||l|l|}
\multicolumn{1}{c}{ Approximating space } & Rate \\
\hline$\tilde{M}_{0}(1, h)$ & 2.00 \\
\hline$\tilde{M}_{0}(2, h)$ & 1.94 \\
\hline
\end{tabular}

Table 1: Experimental rates

\section{References}

[1] J. H. Bramble and S. Hilbert, Estimation of linear functionals on Sobolev. spaces with applications to Fourier transforms and spline interpolation, SIAM J. Numer. Anal., v. 7, pp. 113-124 (1970).

[2] C. N. Dawson, T. F. Russell, and M. F. Wheeler, Some improved error estimates for the modified method of characteristics, to appear in SINUM.

[3] T. Dupont, Galerkin methods for first-order hyperbolic equations: an example, SIAM J. Numer. Anal., v. 10, pp. 890-899 (1970).

[4] J. Douglas, Jr. and T. F. Russell, Numerical methods for convection-dominated diffusion problems based on combining the method of characteristics with finite element or finite difference procedures, SIAM J. Numer. Anal. 19, pp. 871-885 (1982).

[5] R. E. Ewing, T. F. Russell, and M. F. Wheeler, Convergence analysis of an approximation of miscible displacement in porous media by mixed finite elements and a modified method of characteristics, Computer Methods in Applied Mechanics and Engineering, v. 47, pp. 73-92 (1984).

[6] K. W. Morton, A. Priestley, and E. Süli, Stability analysis of the LagrangeGalerkin method with non-exact integration, Report \# 86/14, Oxford University Computing Laboratory (1986).

[7] T. F. Russell, Time-stepping along characteristics with incomplete iteration for a Galerkin approximation of miscible displacement in porous media, SIAM J. Numer. Anal., v. 22, pp. 970-1013 (1985). 